\title{
Metabolomics: A Tool for Anticancer Lead-Finding from Natural Products
}

Authors

Affiliation
Hye Kyong Kim, Erica G. Wilson, Young Hae Choi, Robert Verpoorte

Section Metabolomics, Institute of Biology, Leiden University, Leiden, Netherlands

\section{Key words \\ - natural product \\ - anticancer \\ - metabolomics \\ new approach \\ - in silico \\ - in situ}

\section{Abstract \\ $\nabla$}

Natural products have been the source of many drugs used in modern therapeutics, and particularly in the case of anticancer drugs, more than $50 \%$ originally came from natural products. Their importance as a source of leads for new drugs therefore cannot be underestimated. However, due to the painstaking way of conventional leadfinding, the attention towards natural products has been deviated in the last decades. A new strategy for the detection of active compounds is necessary to get natural product research out of its stalemate. Metabolomics, with its holistic approach and the possibility it provides for the simultaneous detection of all sorts of metabolites, has the potential to be instrumental for this new

\section{Introduction}

$\nabla$

Natural products and particularly plants have long been a productive source of lead molecules or even drugs themselves. Specifically in the case of anticancer drugs, the fact that more than $50 \%$ of the modern oncological drugs are derived from natural products speaks for itself [1].

The traditional way of finding a lead from natural products is based on bioactivity-guided fractionation. For this approach, it is a prerequisite to have a well-established bioassay system which has high-throughput capacity and reliability. The procedure of finding a new active compound is very painstaking since it takes many separation and isolation steps until an active pure compound is finally obtained from the crude extracts. The final structure elucidation may even be a more difficult task as often very complex structures are found. However, the major problem is not only the process, but rather the fact that the bioassays are usually designed to detect single targets. When, as is commonly the case, the activity is due to a mix- approach. Therefore, this review aims at providing examples that illustrate the possibilities of using metabolomics as a tool to find active compounds from natural products, specifically anticancer drugs. Two different methods, in silico and in situ, have been introduced as possible approaches. Current methods to detect anticancer activity in natural products have been briefly reviewed and compared in the first section, and various applications of metabolomics in cancer research are mentioned as they can provide comprehensive information of cancer metabolites utilized in the in situ approach. Metabolomics will certainly improve the efficiency of lead-finding from natural products and thus reinstate this prolific source of potential anticancer drugs.
Dr. Hye Kyong Kim

Section Metabolomics

Institute of Biology

Leiden University

Einsteinweg 55

2300RA Leiden

The Netherlands

Phone: + 31715274510

Fax: + 31715274511

h.k.kim@chem.leidenuniv.nl ture of compounds and their synergistic effect, it is not easy to find novel leads $[2,3]$.

In the last decades, pharmaceutical companies and researchers diverted their attention from plants and microorganisms, turning instead to combinatorial chemistry. The reason for this change in attitude towards natural product research was not the conviction that there was no potential in this field, but rather disenchantment with the ratio of hits versus effort/time and expense. The problem clearly was the methodology or approach which was used, that was too expensive or time-consuming or both at the same time. A new strategy for the detection of the active compounds is necessary to get natural product research out of its stalemate. The decreasing number of novel drugs every year makes for a renewed interest in developing novel approaches. As other reviews have already suggested [2-5], a new approach to find a lead compound from natural products has to be developed. One of the promising tools for new strategies is the use of metabolomics. Metabolomics has been applied in 
many fields in plant science and natural products chemistry, including the quality control of medicinal plants [6-8], the monitoring of the biochemical response of plants to diverse stress conditions [9-11], and the search for differences between genetically modified plants and controls [12,13], among others. This is possible through the general overview of metabolites provided by the metabolomic analysis of organisms submitted to different conditions and consequently allows a better understanding of the biochemical status of biological systems [14].

Metabolomics of human biofluids or tissues, often referred to as "Metabonomics", has been used for the analysis of the metabolic or physiological state of biological systems to provide reliable diagnosis and markers of diseases or toxicity $[15,16]$.

Metabolites are the end product of gene expression and enzyme activity of organisms. The metabolome thus reflects the state of an ongoing biological situation, and the changes in metabolite concentration and/or composition may describe the actual biochemical status of a system better than genomics and proteomics [14]. Measuring metabolite changes resulting from the response of cells upon treatment (with natural products or pure compounds) may help us not only to monitor the response of the cells but also eventually to detect activities of the treated materials themselves. However, to date, the metabolomic approach is more focused on finding system phenomena while the detection of lead compounds is still in a very premature state. Our intention in this review is to explore the feasibility of using metabolomic analysis in the search for lead compounds from natural sources. Due to the amount of existing literature and the importance of the issue, we have restricted this to anticancer lead-finding.

A general overview of methods which are currently used for anticancer activity, measurement, and metabolomic applications in the cancer research field are summarized in the first two sections.

Among many analytical platforms utilized in metabolomic research, NMR has some important advantages over other techniques, the most prominent of which are the broad spectrum of metabolites detected, ease of quantitation, its reproducibility, and straightforward metabolite identification [17]. Particularly in metabolomic analysis of biofluids, urine, blood, and culture extracts, NMR has an edge over other methods, since it allows the introduction of samples directly into the instrument with very simple sample-preparation procedures [18]. Moreover, solid samples from tissues can be directly analyzed using MAS (Magic angle spinning) NMR [19]. Thus, NMR is considered as a method of choice in metabolomic analysis of biofluids (metabonomics). Metabolomics in cancer research mostly deals with cell cultures and animal tissues for which NMR is undoubtedly the most useful and popular method of analysis, therefore our review will give special attention to the application of NMR-based metabolomics in cancer research.

\section{Current Methods for Anticancer Activity Measurements \\ $\checkmark$}

The initial screening methods used to find anticancer leads in natural products are mostly either cell-based or mechanismbased in vitro assays. Cell-based assays usually evaluate the cytotoxicity of plant extracts on cell lines derived from major human neoplasmas. This type of test is routinely conducted with some of 60 cell lines representing 9 distinct types of tumors, which were established by NCI in 1990 [20]. Thanks to the amount of work dedicated to the identification of molecular targets in these cell lines, mechanism-based assays have been established [21]. The targets that have been detected are at different levels: genes (p53, Ras gene) or RNA levels (tyrosine kinase, phosphatases) and enzyme activity (proteasome inhibition assay, histone deacetylase inhibition assay, DT-diapharose) [22]. Using these in vitro assays, many natural products have been tested and new leads have been identified in the last decades [21,23,24].

These assays are quite simple, relatively fast, robust, and easy to set up but often too permissive, resulting in false-positive or false-negative results [22]. To overcome this problem, attempts have been made to develop multicellular tumor spheroid models which have intermediate complexity between in vivo models and in vitro monolayer cultures rather than monolayer culture models. This model has a great potential as it mimics the structural and functional properties of normal and tumor tissues much better, bridging the gap between cell-based assays and animal studies [25]. However, the application of this model to high-throughput screening in anticancer lead-finding has still to be validated. Notwithstanding, this in vitro assay is designed to select compounds for secondary and more comprehensive in vivo testing to ensure that only a small number of compounds with the best chances of success will make it through to the following, increasingly expensive phases of the drug development process.

There are plenty of reviews which deal with the state-of-the-art methods for detecting anticancer activity in natural products, including, among others, an excellent article by Kinghorn and his team [24,26-30]. To date, more than 85000 compounds have been screened using in vitro assays [22], and judging by scientific journals, these still seem to be the major tools used for this objective.

\section{Application of Metabolomics in Cancer Research \\ $\nabla$}

It is quite obvious that monitoring chemical components of tumor cells can provide a better understanding of the complex biochemical process occurring in a cancer. Malignant cells undergo significant changes in their metabolism including a redistribution of metabolic networks, and these changes can result in a metabolic snapshot which is clearly different from that of normal cells [31]. Metabolomic analysis has been applied in many different fields in cancer research including the detection of biomarkers for diagnosis [32], the monitoring of drug response [33], and treatments or prediction of their toxicity [34]. It should be also strongly emphasized that one of the major benefits of metabolomic analysis is that metabolic profiling can usually be achieved using urine or plasma samples apart from the tumor cell itself, increasing the possibility of carrying out large-scale research in a noninvasive manner [35]. Some of the applications of metabolomic studies of cancer cells or samples obtained from cancer patients are described in the following section.

\section{Diagnosis (Biomarkers)}

Biomarkers are widely used in clinical medicine for the purpose of diagnosis or prediction of disease. Finding a new biomarker or, better still, a quantitative biomarker is of great importance in the development of sensitive and specific tests to detect the presence of malignant tumors. NMR-based metabolomics particularly is considered to be a very fast and noninvasive method for the identification of new biomarkers for clinical diagnosis [36]. Indeed, several metabolites have been detected to be metabolic bio- 
markers of tumors thanks to the application of an NMR-based metabolomic approach. In breast cancer cells, one of the best applications of metabolomics in cancer diagnosis so far, the level of total choline-containing compounds (tCho) was found to be increased, whilst the levels of glycerophosphocholine and glucose were decreased as compared to benign tumors and healthy tissue [37-39]. This result is well reflected in the study by Yang et al. [40], who showed that the metabolic activity of several pathways was increased in breast cancer epithelial cell lines, leading to the upregulation of fatty acid synthesis among other changes. Similarly to breast cancer, prostate cancer cells exhibit a distinct metabolic profile characterized by high tCho and phosphocholine levels, along with an increase in the glycolytic products lactate and alanine $[39,41]$. Another good example is provided by the metabolomic study of liver cancer by Yang et al. [42]. They demonstrated that it was possible to distinguish between cancer patients and healthy volunteers, and even more, they were able to distinguish between patients with hepatocirrhosis and hepatitis from patients with liver cancer, with an HPLC-based analysis of urine samples. In this study, several metabolites having cis-diol structures together with typical nucleotide markers (pseudouridin, dihydrouridine) proved to be useful for the differentiation of patients' status [42].

All studies above have demonstrated that the level of several metabolites appeared to be important biomarkers (quantitative biomarkers) in the differentiation of diseased and normal conditions. On the other hand, these biomarkers can also be used to monitor the efficacy of a treatment, for instance a decrease in the tCho signal in the ${ }^{1} \mathrm{H}$-NMR spectra can be associated to the positive response to chemotherapy or radiation in breast, prostate, and brain tumors. Metabolites which were found to be biomarkers in different tumors are listed in an excellent review on this topic [43].

For a biomarker to be really useful, it is essential for it to be detectable in the early stages of the development of a malignant tumor. In cancer, the early detection is very important since it can greatly increase the chance of total remission. Metabolomic analysis has also proved to be useful for this purpose. Odunsi et al. [44] reported the adoption of a metabolomic approach to discriminate between women with epithelial ovarian cancer (EOC) and healthy controls by analyzing their blood serum samples. ${ }^{1} \mathrm{H}-\mathrm{NMR}$ spectra showed substantial differences between blood samples of the EOC patients and healthy subjects, and the consequent PCA analysis of NMR data showed a clear separation of all sera from the patients with malignant tumors, pre-menopausal women, and patients with benign ovarian disease. This clearly proves that the metabolomic analysis of blood samples has a great potential as a novel strategy for the early detection of epithelial ovarian cancer.

Many of these applications have a weakness consisting in the limited number of samples which were used and there might be sample-to-sample variation. This may prove to be an obstacle for the definition of biomarkers in different tumor types and pathological stages of the tumor. Thus, the first requisite for this application to be generalized is that a sufficient number of samples is analyzed and that the techniques are fully standardized in order to improve the reproducibility of these technologies. Quantitative biomarkers or general pattern recognition of tumor cells or samples from cancer patients would be a good way for diagnosis and prediction of disease.

\section{Toxicity assessment}

Metabolomics is a useful tool to monitor early changes in metabolic pathways and therefore be used to predict an anticancer agent's toxicity at an early stage [34]. There are not that many reports on studies made on this aspect in cancer research, but the use of metabolomics in toxicity assessment of drug treatment has been explored opening the possibility for its application to toxicity prediction in cancer therapy. Robertson et al. [45] evaluated the feasibility of applying this technology to screen the toxicity in male rats exposed to toxic agents. Spectra changes were observed shortly after exposure to toxic agents and during the recovery phases. PCA analysis revealed that the metabolites from the samples of all different toxin-treated rats were well clustered in localized regions, which also identified the treataa ed groups but not the control groups. The results demonstrated that this metabolomic approach could readily distinguish the onset and reversal of toxicity with good agreement with clinical biochemistry.

The early diagnosis of toxicity is very necessary in cancer therapaay. This is the case of anthracyclines, which are known to have a potential cardiotoxic effect [35]. The toxicity is cumulative, dose-related, and irreversible, thus, to monitor the early signs of cardiotoxicity will give an opportunity of early intervention, allowing attenuation or abrogation of the cardiac dysfunction.

\section{Response to treatment}

Perhaps the most important contribution which metabolomics can make to the search for an anticancer lead from natural products is providing the means to monitor the effects of anticancer drugs. Cancer is a highly complex disease and abnormalities appear in several genes, proteins, and biochemical pathways. Thus, monitoring all interconnected cellular networks will provide much more information than the use of single molecular markers. This technology can provide a view of all metabolic changes occurring after exposure to a certain anticancer agent. The resulting data provide a view of perturbations which might occur in a large number of tissues. These results can then be used to decide whether to continue the treatment based on the observation of the effectiveness of the drug and eventually to shed more light on its mechanism of action [46].

The application of ${ }^{1} \mathrm{H}-\mathrm{NMR}$ to the prediction of resistance or response to chemotherapy by a neuroblastoma was reported by Lindskog et al. [33]. Several cytotoxic drugs such as cisplatin, etoposide, and irinotecan were used to treat cell lines with sensitivity to these, and the cells were examined by ${ }^{1} \mathrm{H}-\mathrm{NMR}$ ( $\bullet$ Fig. 1 ). Cytotoxic drug treatment was found to produce an increase in methylene and polyunsaturated fatty acid levels and a decrease in choline levels in drug-sensitive neuroblastoma cells. Methylene/choline ratio correlated with cell death and increased in cisplatin-treated drug sensitive cells but not in drug-resistant cell lines, indicating that this ratio can be used as an indicator of cytotoxic responses. A similar study by Morse et al. [47] was performed on breast tumor models. The treatment with docetaxel, an antimicrotubule agent, decreased phosphocholine (PC) levels significantly and increased glycerophosphocholine (GPC) levels in the cell extracts.

Another interesting application, monitoring P-containing metabolites by ${ }^{31} \mathrm{P}$ NMR, allowed the detection of phospholipid metabolism changes since it allows the quantitative analysis of phosphate metabolites in cancer cells [48]. This provides information concerning key components of energy and phospholipids metabolism. Human colon cancer cells were treated with a novel anti- 


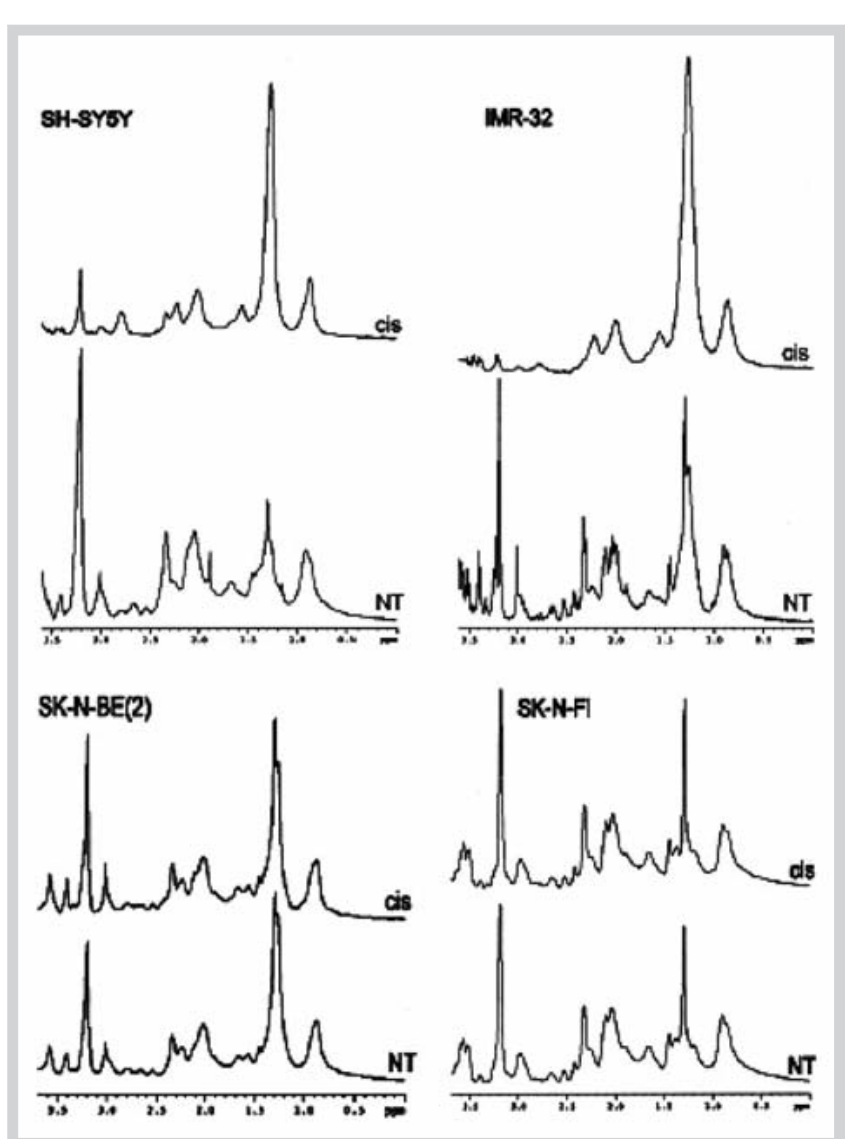

Fig. $1{ }^{1} \mathrm{H}$-NMR spectra of cytotoxic drug-sensitive and -resistant neuroblastoma cell lines. Four neuroblastoma cell lines [drug-sensitive SH-SY5Y and IMR-32 cell lines, drug-resistant phenotypes SK-N-FI, SKN-BE (2)] incubated without (NT) or with cisplatin (cis, $5 \mu \mathrm{M}$ ) for 48 hours. Adapted from [33].

cancer drug: 17-allylamino,17-demethoxygeldanamycin, and ${ }^{31}$ P-NMR spectroscopy allowed the screening of surrogate markers of drug activity. The increased phosphomonoesters/ phosphodiesters ratio was found to correlate with a favorable tumor response, indicating that this ratio can be used as a surrogate marker of response. A similar study was conducted on breast cancer cells by Sterin et al. [49]. Several anticancer agents were tested on the breast cancer cell lines showing different hormonal response and metastatic potential. The ${ }^{31} \mathrm{P}$ NMR spectra of perfused cells revealed a correlation between the mode of action of anticancer drugs and the observed changes in cell metabolic profiles. Briefly, cells treated with antimicrotubule drugs (paclitaxel, vincristine, colchichine) showed a notorious increase in intracellular glycerophosphorylcholine (GPC) while other drugs (methotrexate and doxorubicin) did not affect their levels whatsoever. This study showed that ${ }^{31} \mathrm{P}$-NMR can be used as a diagnostic tool for decisions about treatment of cancers and a new approach to understand the mechanism of action of anticancer drugs. These studies suggest that tumor metabolic profile changes could be helpful in predicting the response to anticancer treatments faster than current methods (e.g., imaging).

Recently, Chung and Griffiths [50] published a review on the characteristic metabolic profiles of cancer cells by NMR after responding to different anticancer treatments with all kinds of drugs from conventional cytotoxic drugs to novel anticancer drugs. This may be very useful to obtain an overview of the different effects of drugs on a cancer cell metabolome.

\section{Metabolomics: Tool for Finding Anticancer Activities \\ $\nabla$}

Metabolomics has been applied in many fields in natural products including the study of the metabolism and biosynthetic pathways under different conditions [14,51]. However, so far, its potential in the detection of active compounds (new leads) still remains untapped. Recently several attempts have been made [6,52-54], and these have shown the feasibility of using metabolomics for this purpose.

Detecting activities in combination with metabolomics can be performed using two different approaches, in silico and in situ. Using the in silico approach a correlation between two different data sets - e.g., metabolomics and bioactivity results - is sought with the help of bioinformatics. On the other hand, the in situ approach consists in the monitoring of the metabolic changes of systems, e.g., tumor cells, microorganism cultures, upon the treatment with tested materials (plant extracts or single pure compound).

Even though most applications reported so far $[6,52,53]$ do not refer to anticancer drug research, there is no reason not to adapt this method to this purpose. Therefore, the techniques/approaches which have been applied in other fields of natural products lead-finding will be reviewed.

In silico approach - correlation by chemometric methods A good example of the successful application of this approach can be found in a recently published paper on the metabolomic study of Galphimia glauca [54]. Galphimia glauca is a Mexican plant which has been used in traditional medicine for the treatment of central nervous disorders. Six different collections of this plant from the whole Mexican area showed different degrees of sedative and anxiolytic activities when tested on animal models. Only two collections were highly active and the others did not show significant activity. Metabolic profiling of six collections was performed using ${ }^{1} \mathrm{H}-\mathrm{NMR}$ and analyzed further by partial least square-discriminant Analysis (PLS-DA) using previous information on their activities ( Fig. 2). Interpretation of the PLS-DA loading plot clearly demonstrated that the signal strongly correlated with the above-mentioned activities, and this signal was identified as corresponding to galphimine ( $\bullet$ Fig. 3 ). When targeted HPLC analysis was performed, it was proven that the two collections possessing strong activities contained a high amount of galphimine while both other (less active) samples did not.

Before this application, Roos et al. [6] had attempted to correlate metabolomes of St. John's wort extract with opioid receptor binding activity. In this study, they showed that NMR combined with multivariate data analysis could effectively predict the activity of preparations, based on a database they constructed. This was suitable for the quality control of preparations made of this plant since it provided a correlation with its efficacy, but they did not attempt to identify the corresponding active compound/s.

In these applications, the use of an appropriate chemometric tool is very critical since it determines the main output from the huge data sets. One of the useful methods to correlate two different data sets (even two different types of "omics" data) is the PLS/OPLS regression method. PLS is a method for relating two data matrices, $\mathrm{X}$ and $\mathrm{Y}$, by a linear multivariate model, but goes beyond traditional regression in that it also models the structure of two data 


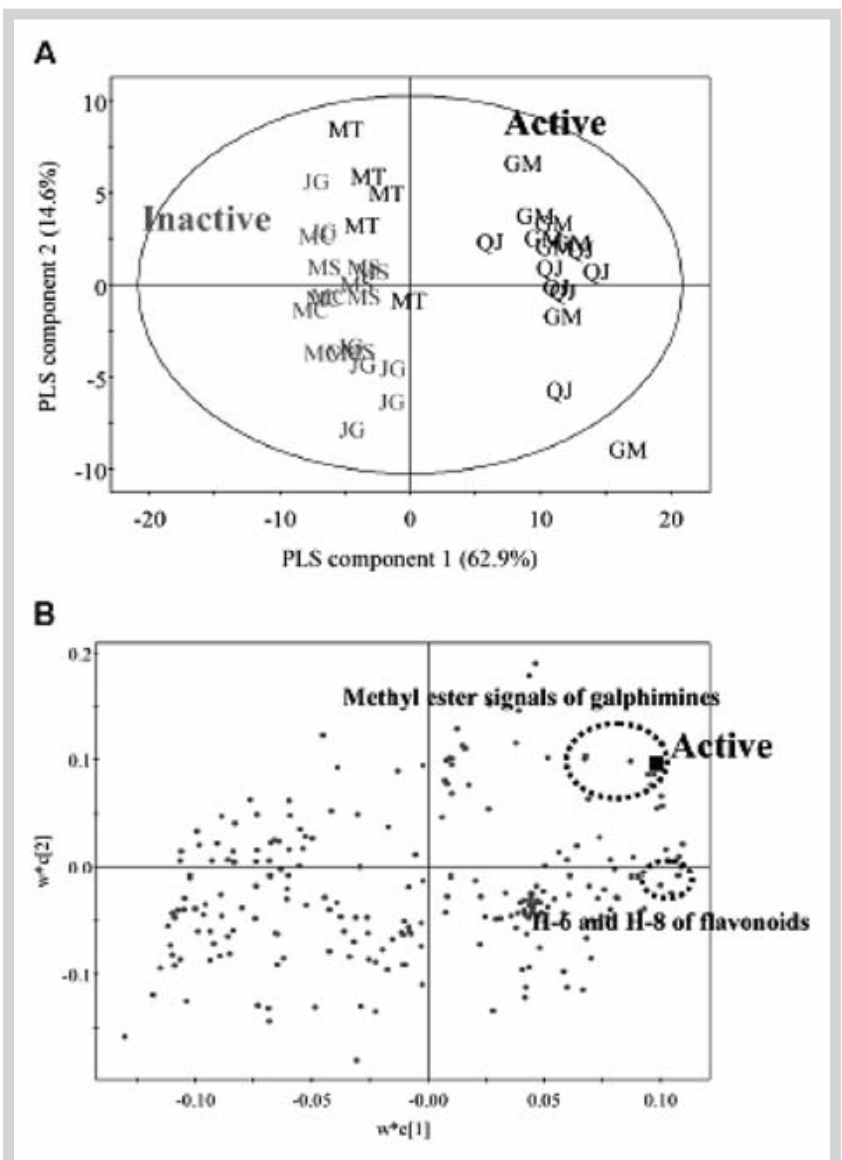

Fig. 2 Score (A) and loading (B) plots of PLS-DA models (PLS component 1 vs. PLS component 2) based on ${ }^{1} \mathrm{H}$-NMR resonances and sedative activity of G. glauca crude extracts based on two classes, active and inactive plants. Active plant materials include $\mathrm{GM}, \mathrm{MT}$, and QJ. Inactive plant materials include MS, MC, and JG. Adapted from [54].

sets. It provides an approach to the quantitative modeling of the often complicated relationships between predictors, $\mathrm{X}$ (metabolome), and responses, Y (bioactivity) [55,56].

On the other hand, the OPLS method is designed to separate the variance of the data matrix $\mathrm{X}$ according to the variance of the data matrix Y, into three parts: the first part represents the variance that is related to $\mathrm{Y}$, the second part, the interfering systematic variation not related (orthogonal) to $\mathrm{Y}$, and the last part contains some residual variance not interfering with the prediction of Y. OPLS is an extension of PLS and has similar objectives to orthogonal signal correction but is integrated directly in the modeling, which allows an easier validation of orthogonal components [57]. The direct correlation and PLS-based approaches were also adopted by Rantalainen et al. [58] in an integrated analysis of NMR metabolic profiles and 2-D differential in gel electrophoresis (DIGE) proteomic data from a murine cancer xenograft model. Metabolites strongly correlating positively or negatively to proteins can be determined in this way. Other useful methods that can be used for this purpose are well described in the review $[57,59]$.

It has to be noted that in such correlations between activity in in vivo assays and the metabolome of extracts, the compounds found to correlate to activity not necessarily are active in itself, they could also be pro-drugs or take part in synergistic activity. This is in fact a major advantage of this approach if compared with the classical bioassay-guided isolation of active compounds in which such compounds will not be found.

In situ approach - metabolic footprinting/fingerprinting Numerous different terms have been used for metabolomic analysis, e.g., metabonomic analysis, metabolic profiling, metabolic fingerprinting, and metabolic footprinting [60]. Among these, metabolic footprinting (exometabolomic) is often applied to the measurement of all extracellular metabolites present, for example, in a culture media. The identified compounds are either metabolites which were secreted by the cells into the medium or medium components biochemically transformed by the organism. On the other hand, metabolic fingerprinting measures a large number of intracellular metabolites detected by a selected analytical technique in defined conditions [60]. Due to the fact that the separation of extracellular media is much easier than any other culture media, metabolic footprinting of microorganisms has been amongst the frontier research fields [61]. Even though metabolic footprinting represents only a fraction of the extracellular contents, it has a tight relationship with the intracellular metabolism, thus it can provide important information and a picture of the intracellular metabolic status.

If this technique is to be used to measure the anticancer activity of natural products, the main strategies to study this should be based on the comparison of the metabolic footprint/fingerprint of cancer cells before/after treatment. We have already seen that metabolomic analysis can be used as a tool to monitor the response of anticancer drugs in tumor cells or the patients' response to anticancer treatment. A similar method could be used to monitor activities as their metabolic profiles can be directly compared to those of positive controls. In this way, biomarkers of activity or the pattern of activity could be detected and subsequently could be identified.

This approach has appeared in the work of Zhi Biao-Yi et al. [52], in which the effect of different antibiotics with different modes of action on the microbial metabolomics was studied. Their results allowed them to conclude that dihydrocucurbitacin F-25-O-acetate, a major constituent of the Chinese plant Hemsleya pengxianensis, showed antimicrobial activity. The metabolome of a Staphylococcus aureus culture treated with a plant extract, dihydrocucurbitacin F-25-0-acetate, and several known antibiotics (streptomycin, vancomycin, erythromycin, rifampicin, etc.) were compared. PCA analysis revealed that dihydrocucurbitacin F-25O-acetate was the component responsible for the main antimicrobial activity on Staphylococcus aureus in $H$. pengxianensis through its ability to inhibit cell wall synthesis, as in the case of vancomycin (๑ Fig. 4).

Similar studies aimed at seeking to discover the mode of action behind antibacterial [53], antifungal [62], and anti-inflammatory activities have been published [63].

One of the advantages of this approach is that it can also be used to predict the mode of actions and not only to select active samples. In order to find an anticancer agent, it is essential that the metabolic footprint/fingerprint of the target cancer cells is analyzed first so that the metabolic footprint/fingerprint of the same cancer cells after treatment with plant extracts can be compared to detect changes. In NMR-based metabolomic analysis, metadata of two different measurements can be easily compared to find changes if existent. One of the possible ways to achieve this is to subtract the obtained spectra, thus revealing the signals that have changed. For this purpose, HSQC (heteronuclear single quantum coherence) spectroscopy is very useful. In this case, as 


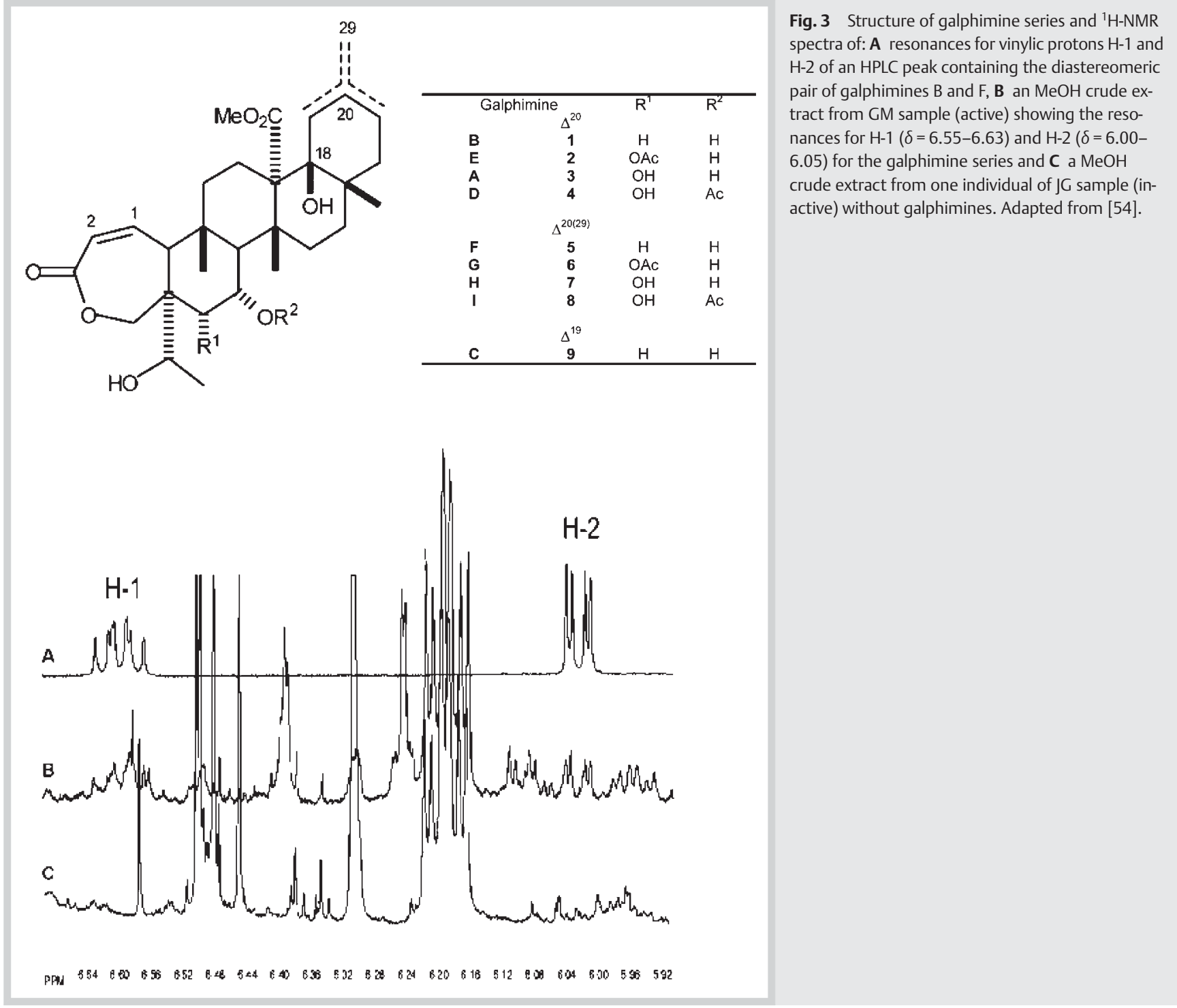

the ${ }^{13} \mathrm{C}$ chemical shift is relatively insensitive to the change of $\mathrm{pH}$ and concentration of samples, it is much more reliable and reproducible to use this rather than ${ }^{1} \mathrm{H}-\mathrm{NMR}$ [64]. Recently this technique was used for the comparison of two different species of Ilex and revealed that the signals of saponins constituted the main difference between the species ( Fig. 5) [65].

In addition, HSQC can be used for a quick screen of the presence of certain metabolites within an extract (a mixture) by comparison of the HSQC spectra of mixtures of known reference compounds [66].

To increase the efficiency of activity screening, the material to be tested from the plant also has to be defined. Usually, the screening material - plant extracts or fractions of extracts - were used for initial testing. In conventional extraction methods, plants or parts of plants are extracted with organic solvent/s, and dried extracts are subjected to screening. Depending on the properties of extraction solvent, the metabolome of the extract will vary, and consequently its activity will surely also differ. It is even possible that some methods may not extract the active metabolites efficiently resulting in low concentrations which may not be sufficient to produce any desirable cellular effect. To improve the potential of finding active compounds and at the same time to reduce elaborated fractionation steps, alternative fast extraction

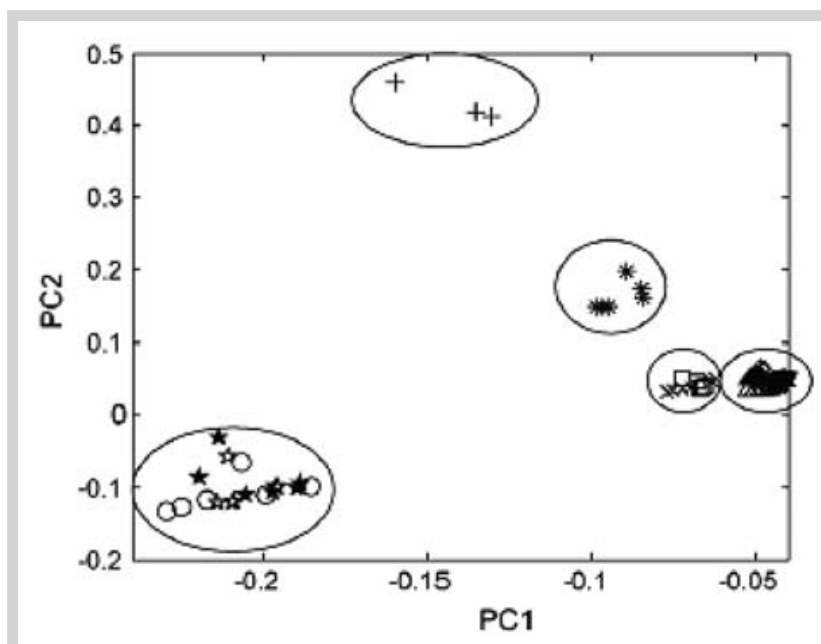

Fig. 4 PCA scatter plot of HPLC profile of controls and cultures treated with different drugs and extract of Hemsleya pengxianensis W.J. Chang [controls $(+)$; norfloxacin $(\times)$; acheomycin $(\diamond)$; lincolmensin $(\mathbf{\Delta})$; cefataxime $\left({ }^{*}\right)$; vancomycin $(\bigcirc)$; rifampicin $(\square)$; erythromycin $(\Delta)$; chloromycetin $(\nabla)$; streptomycin ( $\mathbf{\nabla})$; dihydrocucurbitacin F-25-O-acetate ( $\star$ ); extract of Hemsleya pengxianensis (ઐ)]. Adapted from [52]. 


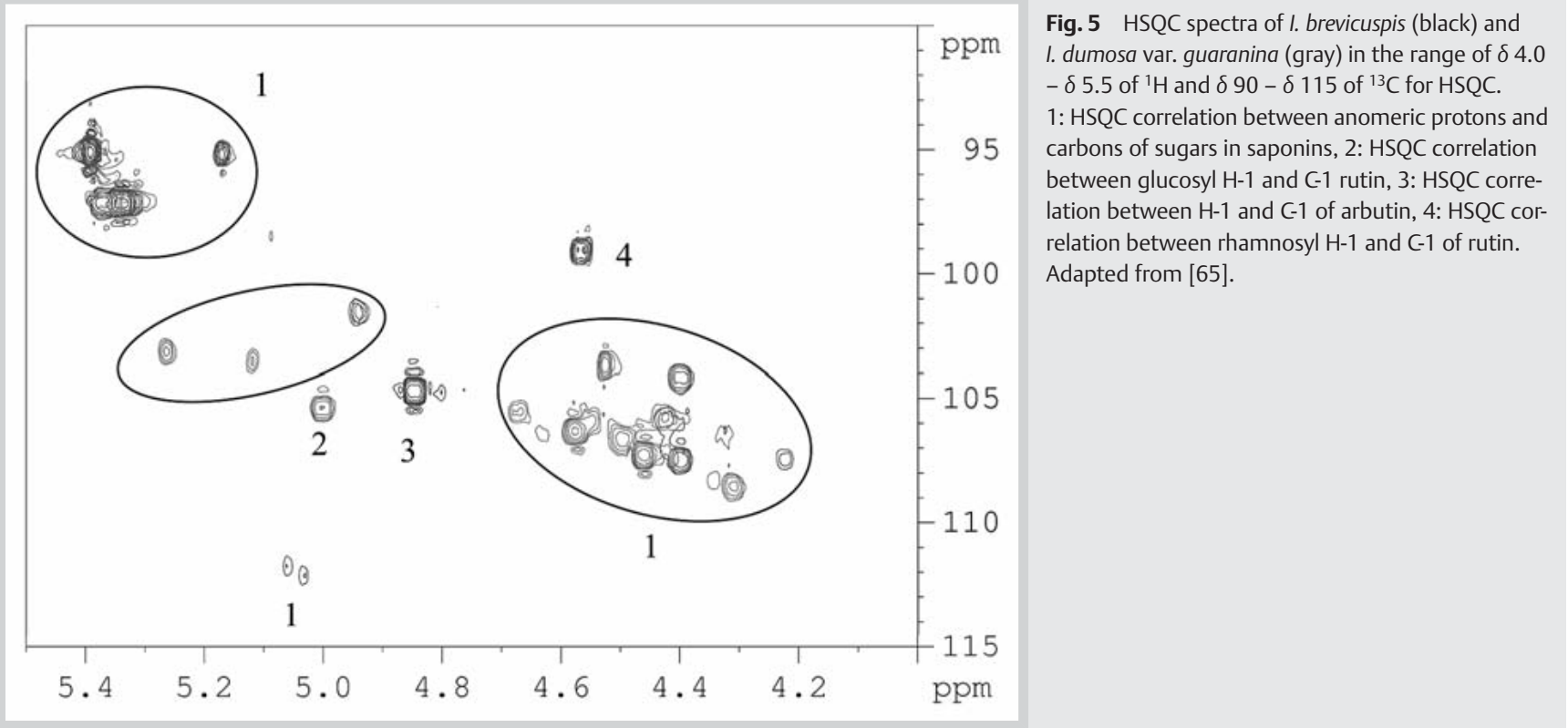

methods should also be considered. This can be accomplished by combining different solvents in one system, applying gradients of polarity and extracting them consecutively in an on-line system. This approach has been developed in our lab and has proved to be very successful allowing the extraction of metabolites ranging from very nonpolar to polar compounds from one single plant sample (unpublished results).

\section{Perspectives \\ $\nabla$}

Metabolomics is a very promising tool to use for the detection of activities from natural products as it has been used in many similar fields, allowing the accumulation of vast experience. What should not be underestimated from the start, is the importance of the interpretation of the biological information revealed by this powerful technique. Therefore, the first requirement is that the obtained data are reliable and reproducible. As many researchers have mentioned, drawing conclusions on the basis of small experiments is very risky [46]. To obtain reliable information from an experiment, a sufficient number of samples have to be analyzed. Furthermore, the communication with metadata obtained from other experiments or groups is only possible if the firm standardization of all processes, from the sample preparation to the measurement and data analysis, needs to be done beforehand. At the same time, if all data have been measured under the same conditions, particularly NMR data can be stored forever and whenever new data is generated, this can be included for use in a new data analysis.

Another important aspect to consider is the assay system required for this approach which has to be fast and simple and suitable to be applied to high-throughput screening. An NMR-based technique - but also other techniques which are used in the metabolomic field - has a great potential for a high-throughput application. Some obstacles are still present, namely the need for fast metabolite identification. The improvements made to NMR sensitivity tend to make fast identification feasible, particularly in mixtures. On the other hand, the existence of public databases of metabolites will again contribute to their identification.
Lastly, whichever compound determined in principle to be active will still have to be submitted to testing on a more solid model system in order to confirm this activity. However, this feature can improve the efficiency and provide shortcuts in the bioassay guided lead-finding research from natural products, thus recovering this prolific source of potential anticancer drugs. Moreover, it can offer a chance to reveal the complex mechanism and synergism of metabolites.

\section{References}

1 Newman DJ, Cragg GM. Natural products as sources of new drugs over the last 25 years. J Nat Prod 2007; 70: 461-477

2 Verpoorte R, Choi YH, Kim HK. Ethnopharmacology and systems biology: a perfect holistic match. J Ethnopharmacol 2005; 100: 53-56

3 Ulrich-Merzenich G, Zeitler H, Jobst D, Panek D, Vetter H, Wagner H. Application of the "-omic-" technologies in phytomedicine. Phytomedicine 2007; $14: 70-82$

4 Rochfort S. Metabolomics reviewed: a new "omic" platform technology for systems biology and implications for natural products research. J Nat Prod 2005; 68: 1813-1820

5 Craige Trenerry V, Rochfort SJ. Natural products research and metabolomics. In: Mander L, Liu H-W, editors. Comprehensive natural products II: chemistry and biology. Amsterdam: Elsevier; 2010: 595-628

6 Roos G, Röseler C, Berger-Büter K, Simmen U. Classification and correction of St. John's wort extracts by nuclear magnetic resonance spectroscopy, multivariate data analysis and pharmacological activity. Planta Med 2004; 70: 771-777

$7 \mathrm{Kim}$ HK, Choi YH, Erkelens C, Lefeber AWM, Verpoorte R. Metabolic fingerprinting of Ephedra species using ${ }^{1} \mathrm{H}-\mathrm{NMR}$ spectroscopy and principal component analysis. Chem Pharm Bull 2005; 53: 105-109

8 Yang SY, Kim HK, Lefeber AWM, Erkelens C, Angelova N, Choi YH, Verpoorte $R$. Application of two-dimensional nuclear magnetic resonance spectroscopy to quality control of ginseng commercial products. Planta Med 2006; 72: 364-369

9 Choi YH, Tapias EC, Kim HK, Lefeber AWM, Erkelens C, Verhoeven JTJ, Brzin J, Zel J, Verpoorte R. Metabolic discrimination of Catharanthus roseus leaves infected by phytoplasma using ${ }^{1} \mathrm{H}-\mathrm{NMR}$ spectroscopy and multivariate data analysis. Plant Physiol 2004; 135: 2398-2410

10 Liang Y-S, Choi YH, Kim HK, Linthorst HJM, Verpoorte R. Metabolomic analysis of methyl jasmonate treated Brassica rapa leaves by two dimensional NMR spectroscopy and multivariate analysis. Phytochemistry 2006; 67: 2503-2511

11 Leiss KA, Choi YH, Abdel-Farid IB, Verpoorte R, Klinkhamer PGL. NMR metabolomics of thrips (Frankliniella occidentalis) resistance in Senecio hybrids. J Chem Ecol 2009; 35: 219-229 
12 Choi HK, Choi YH, Verberne M, Lefeber AWM, Erkelens C, Verpoorte R. Metabolic fingerprinting of wild type and transgenic tobacco plants by ${ }^{1} \mathrm{H}$ NMR and multivariate analysis technique. Phytochemistry 2004; 65: 857-864

13 Le Gall G, Metzdorff SB, Pedersen J, Bennett RN, Colquhoun IJ. Metabolite profiling of Arabidopsis thaliana (L.) plants transformed with an antisense chalcone synthase gene. Metabolomics 2005; 1: 181-198

14 Sumner LW, Mendes P, Dixon RA. Plant metabolomics: large-scale phytochemistry in the functional genomics era. Phytochemistry 2003; 62: 817-836

15 Nicholson JK, Lindon JC, Holmes E. 'Metabonomics': understanding the metabolic responses of living systems to pathophysiological stimuli via multivariate statistical analysis of biological NMR spectroscopic data. Xenobiotica 1999; 29: 1181-1189

16 Nicholson JK, Lindon JC. Systems biology - metabonomics. Nature 2008; 455: 1054-1056

17 Verpoorte R, Choi YH, Mustafa NR, Kim HK. Metabolomics: back to basics. Phytochem Rev 2008; 7: 525-537

18 Beckonert O, Keun HC, Ebbels TMD, Bundy J, Holmes E, Lindon JC, Nicholson JK. Metabolic profiling, metabolomic and metabonomic procedures for NMR spectroscopy of urine, plasma, serum and tissue extracts. Nat Protoc 2007; 2: 2692-2703

19 Broberg A, Kenne L. Use of high-resolution magic angle spinning nuclear magnetic resonance spectroscopy for in situ studies of low-molecular-mass compounds in red algae. Anal Biochem 2000; 284: $367-$ 374

20 Suggitt M, Bibby MC. 50 years of preclinical anticancer drug screening: Empirical to target-driven approaches. Clin Cancer Res 2005; 11: $971-$ 981

21 Gibbs JB. Mechanism-based target identification and drug discovery in cancer research. Science 2000; 287: 1969-1972

22 Damia G, D'Incalci M. Contemporary pre-clinical development of anticancer agents - what are the optimal preclinical models? Eur J Cancer 2009; 45: 2768-2781

23 Holbeck SL. Update on $\mathrm{NCI}$ in vitro drug screen utilities. Eur J Cancer 2004; 40: 785-793

24 Kinghorn AD, Farnsworth NR, Soejarto DD, Cordell GA, Swanson SM, Pezzuto JM, Wani MC, Wall ME, Oberlies NH, Kroll DJ, Kramer RA, Rose WC, Vite GD, Fairchild CR, Peterson RW, Wild R. Novel strategies for the discovery of plant-derived anticancer agents. Pharm Biol 2003; 41: 53-67

25 Lin RZ, Chang HY. Recent advances in three-dimensional multicellular spheroid culture for biomedical research. Biotechnol J 2008; 3: 1172 1184

26 Kinghorn $A D$, Farnsworth NR, Beecher CWW, Soejarto DD, Cordell GA, Pezzuto JM, Wall ME, Wani MC, Brown DM, O'Neill MJ, Lewis JA, Besterman JM. Novel strategies for plant-derived anticancer agents. Int J Pharmacognosy 1995; 33: 48-58

27 Kinghorn AD, Farnsworth NR, Soejarto DD, Cordell GA, Pezzuto JM, Udeani GO, Wani MC, Wall ME, Navarro HA, Kramer RA, Menendez AT, Fairchild CR, Lane KE, Forenza S, Vyas DM, Lam KS, Shu Y-S. Novel strategies for the discovery of plant-derived anticancer agents. Pure Appl Chem 1999: $71: 1611-1618$

28 Kinghorn $A D$, Farnsworth NR, Soejarto DD, Cordell GA, Swanson SM, Pezzuto JM, Wani MC, Wall ME, Oberlies NH, Kroll DJ, Kramer RA, Rose WC, Vite GD, Fairchild CR, Peterson RW, Wild R. Novel strategies for the discovery of plant-derived anticancer agents. Pharm Biol 2003; 41: 53-67

29 Kinghorn AD, Su BN, Jang DS, Chang LC, Lee D, Gu JQ Carcache-Blanco EJ, Pawlus AD, Lee SK, Park EJ, Cuendet M, Gills JJ, Bhat K, Park HS, MataGreenwood E, Song LL, Jang M, Pezzuto JM. Natural inhibitors of carcinogenesis. Planta Med 2004; 70: 691-705

30 Balunas MJ, Kinghorn AD. Drug discovery from medicinal plants. Life Sci 2005; 78: 431-441

31 Boros LG, Brackett DJ, Harrigan GG. Metabolic biomarker and kinase drug target discovery in cancer using stable isotope-based dynamic metabolic profiling (SIDMAP). Curr Cancer Drug Targets 2003; 3: 445-453

32 Brindle JT, Antti H, Holmes E, Tranter G, Nicholson JK, Bethell HWL, Clarkec S, Schofield PM, McKilligin E, Mosedale DE, Grainger DJ. Rapid and non-invasive diagnosis of the presence and severity of coronary heart disease using ${ }^{1} \mathrm{H}-\mathrm{NMR}$-based metabonomics. Nat Med 2002; 8: 1439 1444

33 Lindskog M, Spenger C, Jarvet J, Gräslund A, Kogner P. Predicting resistance or response to chemotherapy by proton magnetic resonance spectroscopy in neuroblastoma. J Natl Cancer Inst 2004; 96: 14571466
34 Robertson DG. Metabonomics in toxicology: a review. Toxicol Sci 2005; 85: 809-822

35 Leo AD, Claudino W, Colangiuli D, Bessi S, Pestrin M, Biganzoli L. New strategies to identify molecular markers predicting chemotherapy activity and toxicity in breast cancer. Ann Oncol 2007; 18: 8-14

36 Lindon JC, Holmes E, Nicholson JK. Metabonomics and its role in drug development and disease diagnosis. Expert Rev Mol Diagn 2004; 4: 189-199

37 Gribbestad IS, Sitter B, Lundgren S, Krane J, Axelson D. Metabolite composition in breast tumors examined by proton nuclear magnetic resonance spectroscopy. Anticancer Res 1999; 19: 1737-1746

38 Sitter B, Lundgren S, Bathen TF, Halgunset J, Fjosne HE, Gribbestadl IS. Comparison of HR MAS MR spectroscopic profiles of breast cancer tissue with clinical parameters. NMR Biomed 2006; 19: 30-40

39 Spratlin JL, Serkova NJ, Eckhardt SG. Clinical applications of metabolomics in oncology: a review. Clin Cancer Res 2009; 15: 431-440

40 Yang C, Richardson AD, Smith JW, Osterman A. Comparative metabolomics of breast cancer. Pac Symp Biocomput 2007: 181-192

41 Swanson MG, Zektzer AS, Tabatabai ZL, Simko J, Jarso S, Keshari KR, Schmitt L, Carroll PR, Shinohara K, Vigneron DB, Kurhanewicz J. Quantitative analysis of prostate metabolites using ${ }^{1} \mathrm{H}$ HR-MAS spectroscopy. Magn Reson Med 2006; 55: 1257-1264

42 Yang J, Xu G, Zheng Y, Kong H, Pang T, Lva S, Yang Q. Diagnosis of liver cancer using HPLC-based metabonomics avoiding false-positive result from hepatitis and hepatocirrhosis diseases. J Chromatogr B 2004; 813: 59-65

43 Griffin JL, Shockcor JP. Metabolic profiles of cancer cells. Nat Rev Cancer 2004; 4: 551-561

44 Odunsi K, Wollman RM, Ambrosone CB, Hutson A, McCann SE, Tammela J, Geisler JP, Miller G, Sellers T, Cliby W, Qian F, Keitz B, Intengan M, Lele S, Alderfer JL. Detection of epithelial ovarian cancer using ${ }^{1} \mathrm{H}-\mathrm{NMR}$-based metabonomics. Int J Cancer 2005; 113: 782-788

45 Robertson DG, Reily MD, Sigler RE, Wells DF, Paterson DA, Braden TK. Metabonomics: Evaluation of nuclear magnetic resonance and pattern recognition technology for rapid in vivo screening of liver and kidney toxicants. Toxicol Sci 2000; 57: 326-337

46 Claudino WM, Quattrone A, Biganzoli L, Pestrin M, Bertini I, Di Leo A. Metabolomics: available results, current research projects in breast cancer, and future applications. J Clin Oncol 2007; 25: 2840-2846

47 Morse DL, Raghunand N, Sadarangani P, Murthi S, Job C, Day S, Howison C, Gillies RJ. Response of choline metabolites to docetaxel therapy is quantified in vivo by localized ${ }^{31} \mathrm{P}$ MRS of human breast cancer xenografts and in vitro by high-resolution ${ }^{31} \mathrm{P}$ NMR spectroscopy of cell extracts. Magn Reson Med 2007; 58: 270-280

48 Negendank WG. Studies of human tumors by MRS: a review. NMR Biomed 1992; 5: 303-324

49 Sterin M, Cohen JS, Mardor Y, Berman E, Ringel I. Levels of phospholipids metabolites in breast cancer cells treated with antimitotic drugs: a ${ }^{31} \mathrm{P}-$ magetic resonance spectroscopy study. Cancer Res 2001; 61: 75367543

50 Chung YL, Griffiths JR. Using metabolomics to monitor anticancer drugs. In: Kroemer G, Mumberg D, Keun K, Riefke B, Steger-Hartmann T, Petersen K, editors. Oncogenes meet metabolism. Ernst Schering Foundation Symposium Procedings. Heidelberg: Springer-Verlag; 2008: 55-78

51 Dixon RA, Sumner LW. Legume natural producs: understanding and manipulating complex pathways for human and animal health. Plant Physiol 2003; 131: 878-885

52 Zhi Biao-Yi, Yan Yu Y, Yi Zeng-Liang. Investigation of antimicrobial model of Hemsleya pengxianensis W.J. Chang and its main active component by metabolomics technique. J Ethnopharmacol 2008; 116: 89-95

53 Liu Y, Wen J, Wang Y, Li Y, Xu W. Postulating modes of action of compounds with antimicrobial activities through metabolomics analysis. Chromatographia 2010; 71: 253-258

54 Cardoso-Taketa AT, Pereda-Miranda R, Choi YH, Verpoorte R, Villarreal $M L$. Metabolic profiling of the Mexican anxiolytic and sedative plant Galphimia glauca using nuclear magnetic resonance spectroscopy and multivariate data analysis. Planta Med 2008; 74: 1295-1301

55 Wold S, Sjöström M, Eriksson L. PLS-regression: a basic tool of chemometrics. Chemometrics Intell Lab Syst 2001; 58: 109-130

56 Holmes $\mathrm{E}$, Antti $\mathrm{H}$. Chemometric contributions to the evolution of metabonomics: mathematical solutions to characterizing and interpreting complex biological NMR spectra. Analyst 2002; 127: 1549-1557

57 Trygg J, Wold S. O2-PLS, a two-block (X-Y) latent variable regression (LVR) method with an integrated OSC filter. J Chemom 2003; 17: 53-64 
58 Rantalainen M, Cloarec O, Beckonert O, Wilson ID, Jackson D, Tonge R, Rowlinson R, Rayner S, Nickson J, Wilkinson RW, Mills JD, Trygg J, Nicholson JK, Holmes E. Statistically integrated metabonomic-proteomic studies on a human prostate cancer xenograft model in mice. J Proteom Res 2006; 5: 2642-2655

59 Ebbels TMD, Rachel Cavill R. Bioinformatic methods in NMR-based metabolic profiling. Prog Nucl Magn Reson Spectr 2009; 55: 361-374

60 Dunn WB. Current trends and future requirements for the mass spectrometric investigation of microbial, mammalian and plant metabolomes. Phys Biol 2008; 5: 1-24

61 Kell DB, Brown M, Hazel M, Davey HM, Dunn WB, Spasic I, Oliver SG. Metabolic footprinting and systems biology: the medium is the message. Nat Rev Microbiol 2005; 3: 557-565

62 Allen J, Davey HM, Broadhurst D, Rowland JJ, Oliver SG, Kell DB. Discrimination of modes of action of antifungal substances by use of metabolic footprinting. Appl Environ Microbiol 2004; 70: 6157-6165
63 Verhoeckx KCM, Bijlsma S, Jespersen S, Ramaker R, Verheij ER, Witkamp $R F$, van der Greef J, Rodenburg RJT. Characterization of anti-inflammatory compounds using transcriptomics, proteomics, and metabolomics in combination with multivariate data analysis. Int Immunopharmacol 2004; 4: 1499-1514

64 Xi Y, De Ropp JS, Viant MR, Woodruff DL, Yu P. Improved identification of metabolites in complex mixtures using HSQC NMR spectroscopy. Anal Chim Acta 2008; 614: 127-133

65 Kim HK, Saifullah, Khan S, Wilson EG, Prat Kricun SD, Meissner A, Goraler $S$, Deelder AM, Choi YH, Verpoorte R. Metabolic classification of South American Ilex species by NMR-based metabolomics. Phytochemistry 2010; 71: 773-784

66 Lewis IA, Schommer SC, Hodis B, Robb KA, Tonelli M, Westler WM, Sussman MR, Markley JL. Method for determining molar concentrations of metabolites in complex solutions from two-dimensional ${ }^{1} \mathrm{H}^{-13} \mathrm{C}$ NMR spectra. Anal Chem 2007; 79: 9385-9390 